\title{
Prognostic efficacy of cardiac biomarkers for mortality in dialysis patients
}

\author{
P. E. Hickman, ${ }^{1,2}$ D. A. McGill,, ${ }^{3,4}$ G. Talaulikar, ${ }^{4,5}$ B. Hiremagalur, ${ }^{4,5}$ J. Bromley, ${ }^{5}$ A. Rahman, ${ }^{3}$ G. Koerbin, ${ }^{1}$ \\ E. Southcott ${ }^{1}$ and J. M. Potter ${ }^{1,2}$ \\ Departments of ${ }^{1}$ Chemical Pathology, ${ }^{3}$ Cardiology and ${ }^{5}$ Nephrology, The Canberra Hospital and Departments of ${ }^{2}$ Pathology and \\ ${ }^{4}$ Medicine, ANU Medical School, Canberra, Australian Capital Territory, Australia
}

\author{
Key words \\ renal failure, dialysis, NT-pro-BNP, BNP, \\ troponin, mortality.

\section{Correspondence} \\ Peter E. Hickman, ACT Pathology and ANU \\ Medical School, The Canberra Hospital, \\ Yamba Drive, Garran, ACT 2605, Australia. \\ Email: peter.hickman@act.gov.au \\ Received 4 July 2008; accepted \\ 23 September 2008.
}

doi:10.1111/j.1445-5994.2009.01846.x

\begin{abstract}
Background: The high prevalence of cardiovascular mortality in the endstage renal disease population is well established. The aim of this current study was to document the relative prognostic significance of established cardiac biomarkers troponin $\mathrm{T}(\mathrm{TnT})$, troponin I (TnI), B-type natriuretic peptide (BNP) and N-terminal pro-BNP (NT-pro-BNP) in this population.

Methods: A prospective cohort study of dialysis patients undertaken in a single tertiary centre in Australia. Relevant clinical and biochemical information was collected at entry and all patients followed up prospectively without any loss to follow up. End-point of interest was all-cause mortality. Statistical analysis using Cox proportional hazards was used to study relationship between competing covariates and outcome. A total of 143 patients with a mean age of $59.67 \pm 15.49$ years was followed up for a median duration of 30 months. Of these patients, 89.3\% were white Australians of European ancestry. Twenty-seven per cent had an established diagnosis of diabetes mellitus. The mean concentrations $( \pm S D)$ of TnT, TnI, BNP and N-terminal peptide pro-BNP (NT-pro-BNP) were $0.08 \pm 0.04 \mu \mathrm{g} / \mathrm{L}, \quad 0.09 \pm 0.2 \mu \mathrm{g} / \mathrm{L}$, $270 \pm 117 \mathrm{ng} / \mathrm{L}$ and $1434 \pm 591 \mathrm{ng} / \mathrm{L}$ respectively.

Results: Twenty-eight subjects died during the period of follow up. By univariate analysis, all cardiac markers (TnT, TnI, BNP, NT-pro-BNP and C-reactive protein) were significantly associated with an increase in mortality. On Cox proportionate hazards analysis, only albumin and NT-pro-BNP showed a significant association with mortality, with hazard ratios of 0.834 , $95 \%$ confidence interval (CI) $0.779-0.893, P<0.001$, and $1.585,95 \% \mathrm{CI}$ 1.160-20165, $P=0.004$ respectively.

Conclusion: In patients with end-stage renal failure on dialysis NT-pro-BNP provides greater prognostic information compared with TnT and TnI.
\end{abstract}

\section{Introduction}

There is a high morbidity and mortality among patients with renal failure undergoing dialysis. In Australia in

Funding: This work was supported by a grant from the Private Practice Fund of The Canberra Hospital.

Conflict of interest: None.
$2005,14.5 \%$ of all dialysis patients died and $39 \%$ of these deaths were due to cardiac events. ${ }^{1}$ Similar figures are reported from other countries with a high standard of health care. ${ }^{2}$

Several studies have reported that detection of tropo$\operatorname{nin} \mathrm{T}(\mathrm{TnT})$ in the blood of asymptomatic patients on dialysis is a predictor of adverse outcome in this population. $^{3-5}$ More recently similar associations have been shown for other biomarkers including troponin I 
(TnI), ${ }^{6,7}$ highly sensitive C-reactive protein (CRP), B-type natriuretic peptide (BNP) and $\mathrm{N}$-terminal peptide proBNP (NT-pro-BNP). ${ }^{6-9}$ Little information is available on the relative prognostic significance of either BNP or NT-pro-BNP on the background of detectable TnT in the end-stage renal disease population.

We have carried out a prospective study in which blood was collected for baseline investigations from a dialysis population, which was then observed for an average of 2 years. The objectives of the study were to confirm the use of cardiac troponins as useful prognostic biomarkers in our population and also to establish the relative value of measuring TnT or TnI using newer, more sensitive assays. In addition, we planned to investigate the relative prognostic value of the cardiac troponins with other established (CRP and albumin) and emerging (BNP and NT-pro-BNP) biomarkers. The results of the first objective have been published, ${ }^{7}$ and this paper reports the results of the latter investigation.

\section{Methods}

\section{Patient population and study design}

The Canberra Hospital provides dialysis services to patients in the Australian Capital Territory (ACT) and nearby regions of southern New South Wales.

During a 4-week period, all patients undergoing either haemodialysis or peritoneal dialysis were approached and invited to take part in this study. Only one haemodialysis patient declined to take part in the study. Approximately 20 peritoneal dialysis patients did not attend the unit during the recruitment period and did not take part in the study. A total of 143 patients was enrolled of whom 31 were undergoing peritoneal dialysis. Demographic and relevant clinical details were obtained both by direct query of the patients and by examination of the individual patient records. Comorbidities were ascertained using the ANZDATA registry for this population cohort. The design was at baseline a cross-sectional study with a longitudinal follow up (cohort study), with all-cause mortality as the primary end-point. Patients were followed for an average of 2 years (median 30 months). There were no exclusion criteria apart from declining to participate. The study was approved by the ACT Health Human Research Ethics Committee.

Of necessity, the dialysis population maintains a close relationship with the renal unit and this allows for reliable long-term follow up with ascertainment of hard outcomes including death. Most of the patients in the study remained with the same renal unit, but for patients transferred to other units, death was ascertained by telephonic contact with the dialysis nurse managers. The study looked at all-cause mortality so ascertainment bias was not an issue.

\section{Laboratory procedures and analyses}

All blood samples were collected immediately predialysis, into Vacuette tubes (Greiner Bio-one $\mathrm{GmbH}$, Kremsmunster, Austria) containing potassium EDTA for BNP analysis, and lithium heparin with a separator gel for all other assays. Samples were centrifuged at $3000 \mathrm{~g}$ for $10 \mathrm{~min}$ and aliquots for each assay stored at $-70^{\circ} \mathrm{C}$. Before assay each aliquot was thawed and recentrifuged at $3000 \mathrm{~g}$ for $10 \mathrm{~min}$.

Analysis for TnT was carried out on the Roche Elecsys 1010 (Roche Diagnostics Australia, Sydney, Australia), with assay coefficients of variation (CV) being $6.0 \%$ at $0.11 \mu \mathrm{g} / \mathrm{L}$ and $2.5 \%$ at $2.5 \mu \mathrm{g} / \mathrm{L}$. TnI assay was carried out on the Abbott Architect ci8200 (Abbott Diagnostics, Sydney, Australia) (assay CV 8.3\% at $0.12 \mu \mathrm{g} / \mathrm{L}$ and $7.5 \%$ at $0.53 \mu \mathrm{g} / \mathrm{L}$ ). BNP measurement was carried out on the Abbott AxSYM analyser (assay CVs 10.3\% at $108 \mathrm{ng} / \mathrm{L}$ and $8.8 \%$ at $490 \mathrm{ngl} / \mathrm{L}$ ) and NT-pro-BNP measurement was carried out on the Roche Elecsys 1010 analyser with assay CVs being $4.8 \%$ at $148 \mathrm{ng} / \mathrm{L}$ and $2.4 \%$ at $5080 \mathrm{ng} / \mathrm{L}$. CRP was measured on the Abbott Ci8200 analyser with CVs being $8.0 \%$ at $0.70 \mathrm{mg} / \mathrm{L}$ and $1.4 \%$ at $11.80 \mathrm{mg} / \mathrm{L}$.

All assays for individual analytes were carried out at the one time so as to eliminate any inter-assay variability.

\section{Statistical analyses}

We used univariate survival sample size calculations because the multivariate analysis was exploratory, thus: assuming 0.05 as $\alpha$ (the probability of falsely rejecting the null hypothesis); the power required as 0.80 (the probability of correctly rejecting the null hypothesis of equal survival times); an assumed relative risk of 2.5 for exposure to each of TnI, TnT, CRP and the upper quartile level of NT-Pro-BNP (based on past data); a survival time in the 'unexposed' group of 24 months; an accrual time during which patients were recruited of 3 months; additional follow-up time after end of recruitment of 24 months; and a ratio of unexposed (alive) to exposed (dead) patients of 0.25 ; the sample size required was 136 patients. This low sample size is due to the high event rate of $25 \%$ based on previous data, ${ }^{1}$ and the high relative risk, also based on known data.

All relevant clinical and laboratory data were entered onto a spreadsheet and all subsequent statistical analysis was with SPSS version 14 . 
All categorical outcome measures were reported as both an absolute number and proportions. Continuous variables with a normal distribution were expressed as mean and standard deviation (SD), with $95 \%$ confidence intervals (CI). Skewed continuous variables were reported as median with the inter-quartile range (IQR).

Categorical variables were compared by $\chi^{2}$ analysis or Fisher's exact test if any cells were $\leq 5$. Differences between groups for normally distributed variables were analysed with the independent $t$-test. Non-normal distributed continuous variables that were not transformable were compared with non-parametric tests (Mann-Whitney $U$-test).

Long-term survival related to individual variables was analysed in a Kaplan-Meier model with the log-rank test to evaluate any differences. Kaplan-Meier estimates were also used to describe the time-course outcome for the biomarkers in quartiles. The Cox proportional hazard regression model was used to investigate simultaneously the effect of several prognostic variables on survival by calculating the hazard ratio for all biomarkers with adjustment for other variables when clinically indicated or because of a univariate association.

Study validation processes included analyses that would provide logically expected results and analyses for comparison with current published results. The former included a univariate Kaplan-Meier survival analysis for patients with cardiac symptoms, for those with a preexisting cardiac condition and the presence of an abnormality on echocardiography in those patients having the procedure for clinical reasons.

Published cut-off points for the biomarkers were examined. Receiver-operator characteristic (ROC) curve analysis measuring the area under the curve (AUC) was used to enable comparison with previously published results. ${ }^{6}$ The numbers of patients and the frequency of events were too small to allow meaningful assessment of cut-off points for prognostic assessment for individual patients with ROC analysis.

Test accuracy assessments (inter-test and intra-test) were with the coefficient of variation. All estimates of test accuracy and association or differences were accompanied by a standard error of the estimate of accuracy and 95\% CI around the estimate.

All statistical hypothesis tests were assessed with the two-tailed tests with $P<0.05$.

\section{Results}

A follow up of all the 143 patients was carried out for a median duration of 30 months. During this time there were 28 deaths. The population demographics and clinical characteristics of our 143 subjects are shown in
Table 1 Population demographic and clinical characteristics

\begin{tabular}{ll}
\hline & \multicolumn{1}{c}{ Participants ( $n=143)$} \\
\hline Age mean (SD); median (IQR) years & 59.67 (15.49); 63.00 (52-73) \\
Females (\%) & 37 \\
Race (\%) & \\
$\quad$ White & 89.3 \\
Black & 3.6 \\
Asian & 3.6 \\
Other & 3.5 \\
Hypertension diagnosis, $n$ (\%) & $60(42)$ \\
History of cardiac comorbidity, $n$ (\%) & $54(38)$ \\
History of PTCA (\%) & 4 \\
History of CABG (\%) & 11 \\
Time on dialysis, median (IQR, range) & $40.56(30,12-60,1-240)$ \\
$\quad$ (months) & \\
Average session dialysis time (SD) (h) & $4.18(0.59)$ \\
Average sessions per week (SD) & $3.0(0.27)$ \\
Diabetes, $n$ (\%) & $38(27)$ \\
Hypercholesterolaemia, $n$ (\%) (cholesterol & $8(6)$ \\
$\quad>5.5$ mmol/L) & \\
\hline
\end{tabular}

CABG, coronary artery bypass surgery; IQR, inter-quartile range; PTCA, percutaneous transluminal coronary angioplasty; SD, standard deviation.

Tables 1 and 2 shows the means, medians and ranges for the different biomarkers we studied.

Univariate analysis of our categorical data showed many significant associations of objective measures with all-cause mortality as shown in Table 3. Clinical characteristics that showed an association were age, preexisting cardiac comorbidity and presence of cardiac symptoms. Biomarkers showed a much stronger association with all-cause mortality, with albumin, CRP, TnT (both presence and quartiles), TnI (quartiles only), BNP (both above the median and quartiles), NT-pro-BNP (both above the median and quartiles) and CRP all significant with $P<0.005$. Whereas TnI presence did not reach statistical significance, comparison of the upper

Table 2 Biomarker concentrations ${ }^{\dagger}$

\begin{tabular}{lcc}
\hline Biomarkers & Mean (SD) & Median (IQR) \\
\hline $\operatorname{TnT}(\mu \mathrm{g} / \mathrm{L})$ & $0.080(0.128)$ & $0.043(0.011-0.098)$ \\
$\operatorname{Tnl}(\mu \mathrm{g} / \mathrm{L})$ & $0.090(0.52)$ & $0.20(0.01-0.0425)$ \\
CRP $(\mathrm{mg} / \mathrm{L})$ & $15.08(22.95)$ & $7.59(2.87-17.23)$ \\
Albumin $(\mathrm{g} / \mathrm{L})$ & $41.3(5.85)$ & $42.0(39.0-45.0)$ \\
BNP $(\mu \mathrm{g} / \mathrm{L})$ & $270.1(331.2)$ & $116.8(51-328.8)$ \\
NT-pro-BNP $(\mu \mathrm{g} / \mathrm{L})$ & $1434(2934)$ & $591(207-1568)$ \\
Total cholesterol $(\mathrm{mmol} / \mathrm{L})$ & $3.97(0.96)$ & $3.81(3.35-4.51)$ \\
\hline
\end{tabular}

†Samples for biomarker measurement were collected immediately predialysis at the start of the study period. Assay details are to be found in the Methods section. BNP, B-type natriuretic peptide; CRP, C-reactive protein; IQR, inter-quartile range; NT-pro-BNP, N-terminal peptide proBNP; SD, standard deviation. 
Table 3 Frequency associations for all-cause mortality of categorical variables and biomarkers defined in categories at the beginning of the study

\begin{tabular}{|c|c|c|c|}
\hline & Odds ratio ${ }^{\dagger}$ & OR 95\% Cl & Pearson's $\chi^{2}$ (P-value) \\
\hline Age in the highest 50th percentile & 3.77 & $1.48-9.55$ & $8.461(0.004)$ \\
\hline Female compared with male gender & 0.65 & $0.28-1.49$ & $1.044(0.307)$ \\
\hline Pre-existing cardiac comorbidity & 2.63 & $1.14-6.25$ & $5.301(0.021)$ \\
\hline Presence of cardiac symptoms & 4.76 & $1.08-20.00$ & $4.980(0.025)^{*}$ \\
\hline Diabetic of any type & 1.11 & $0.50-2.86$ & $0.044(0.834)$ \\
\hline Insulin-dependent diabetic & 4.48 & $0.84-23.51$ & $3.680(0.055)$ \\
\hline History of a failed transplant & 0.90 & $0.84-0.95$ & $3.189(0.124)^{*}$ \\
\hline Hypertension previously documented & 1.02 & $0.43-2.43$ & $0.001(0.970)$ \\
\hline Albumin $<35$ & 37.04 & $9.54-142.86$ & $48.28(<0.000)$ \\
\hline CRP $>7.1$ & 8.04 & $2.61-24.75$ & $16.51(<0.001)$ \\
\hline CRP upper quartile $e^{\star \star}$ & 4.50 & $1.86-10.91$ & $12.17(<0.001)$ \\
\hline TnT detectable & 11.33 & $1.48-86.79$ & $8.23(0.004)$ \\
\hline TnT upper quartile $e^{\star \star}$ & 4.23 & $1.76-10.14$ & $11.39(0.001)$ \\
\hline Tnl detectable & 6.37 & $0.82-49.58$ & $4.01(0.087)^{\wedge}$ \\
\hline Tnl upper quartile** & 4.50 & $1.86-10.91$ & $12.17(<0.001)$ \\
\hline NT-pro-BNP above median (>591 ng/L) & 4.86 & $1.83-12.91$ & $11.39(0.001)$ \\
\hline NT-pro-BNP upper quartile (>1567 ng/L)** & 5.42 & $2.24-13.15$ & $15.71(<0.001)$ \\
\hline BNP above median (>116.7 ng/L) & 3.09 & $1.26-7.59$ & $6.41(0.011)$ \\
\hline BNP upper quartile $(>270 \mathrm{ng} / \mathrm{L})^{* *}$ & 3.62 & $1.51-8.71$ & $8.91(0.003)$ \\
\hline
\end{tabular}

*Fisher's exact test, ^Yate's continuity correction. ${ }^{\dagger}$ Odds ratio (OR) for being alive rather than dead at the end of the follow-up period with the variable present compared to the variable being absent (OR $<1$ means more likely to be alive and OR $>1$ more likely to have died). ${ }^{* *}$ Compared with lower three quartiles. BNP, B-type natriuretic peptide; CRP, C-reactive protein; NT-pro-BNP, N-terminal pro-BNP; Tnl, troponin I; TnT, troponin T.

quartile versus the lower three quartiles was very strongly positive with $P<0.001$.

When analysis was carried out looking at the association of all-cause mortality with continuous variables (Table 4), the same strong association was observed as shown in Table 3.

There is a significant increase in the frequency of allcause mortality with increasing quartiles for the biomarkers of interest, namely TnT (Pearson's $\chi^{2}=18.641$ $P=0.002$ ); TnI (Pearson's $\chi^{2}=14.934 P<0.001$ ); CRP (Pearson's $\chi^{2}=19.996 P<0.001$ ); albumin (Pearson's $\left.\chi^{2}=22.236 \quad P<0.001\right) ; \quad$ BNP $\quad$ (Pearson's $\chi^{2}=8.730$; $P=0.033$ ); and NT-Pro-BNP (Pearson's $\chi^{2}=18.898$; $P<0.001)$. Using the Kaplan-Meier survival analysis, the biomarkers of interest were significantly associated with all-cause mortality with increasing quartiles (Table 5); for albumin this was with a decrease in the value (Table 5).

All the biomarkers of interest were entered into Cox's regression model, namely albumin, natural log transformed BNP, NT-Pro-BNP, and CRP, with TnT and TnI in quartiles. The albumin concentration (hazard ratio 0.837 , $95 \%$ CI $0.783-0.896, P<0.001)$ and the natural $\log$ of

Table 4 All-cause mortality outcomes for continuous variables

\begin{tabular}{|c|c|c|c|c|}
\hline Variable & Alive mean (SD) & Dead mean (SD) & Mean difference $(95 \% \mathrm{Cl})$ & $P$-value (two tailed) \\
\hline Age at beginning (years)* & $57.70(15.87)$ & $67.75(10.62)$ & $-10.046(-16.30 \text { to }-3.79)^{* *}$ & $<0.001$ \\
\hline Hours per dialysis session* & $4.23(0.63)$ & $3.98(0.29)$ & $0.25(0.07$ to 0.43$)$ & 0.007 \\
\hline BNP $(n g / L) *$ & 222 (292) & 465 (407) & $-243(-406 \text { to }-77)^{\star}$ & 0.005 \\
\hline Natural log BNP* & $4.641(1.329)$ & $0.570(1.321)$ & $-0.929(-1.482$ to -0.375$)$ & 0.001 \\
\hline NT-pro-BNP* & $1117(1821)$ & 3930 (4951) & $-2813(-4758 \text { to }-867)^{\star}$ & 0.006 \\
\hline Natural log NT-pro-BNP* & $6.052(1.425)$ & $7.487(1.469)$ & $-1.435(-2033$ to -813$)$ & $<0.001$ \\
\hline Troponin T*** & $0.065(0.112)$ & $0.141(0.166)$ & & $<0.001$ \\
\hline Troponin 1*** & $0.036(0.113)$ & $0.305(1.115)$ & & $<0.001$ \\
\hline Albumin* & $42.6(4.22)$ & $35.93(8.22)$ & $6.67(3.40 \text { to } 9.94)^{\star \star}$ & $<0.001$ \\
\hline CRP* & $11.86(16.70)$ & $27.74(36.59)$ & $-15.87(-30.86 \text { to }-1.38)^{\star \star}$ & 0.033 \\
\hline Natural log CRP* & $1.76(1.21)$ & $2.68(1.28)$ & $-0.913(-1.426$ to -0.400$)$ & 0.001 \\
\hline Triglyceride levels** & $1.942(1.073)$ & $1.498(0.807)$ & $0.444(0.076$ to 0.813$)$ & 0.019 \\
\hline
\end{tabular}

${ }^{\star} t$-test for independent means, ${ }^{\star *}$ equal variance not assumed, ${ }^{* \star *}$ Mann-Whitney non-parametric test. 
Hickman et al.

Table 5 Kaplan-Meier survival analysis of biological markers in quartiles for all-cause mortality

\begin{tabular}{|c|c|c|c|c|c|}
\hline Biological marker & Quartile 1 & Quartile 2 & Quartile 3 & Quartile 4 & Log-rank (Mantel-Cox) \\
\hline cTnT ( $\mu \mathrm{g} / \mathrm{L})$ & $<0.010$ & $0.011-0.042$ & $0.043-0.097$ & $>0.098$ & \\
\hline Patients, $n$ & 35 & 36 & 36 & 36 & \\
\hline Mortality, $n$ (\% of total dead) & $1(3.6)$ & $6(21.4)$ & $7(25)$ & $14(50)$ & $15.78 P=001$ \\
\hline $\mathrm{cTnl}(\mu \mathrm{g} / \mathrm{L})$ & $<0.010$ & $0.011-0.020$ & $0.021-0.043$ & $>0.043$ & \\
\hline Patients, $n$ & 34 & 35 & 35 & 34 & \\
\hline Mortality, $n$ (\% of total dead) & $1(3.6)$ & $3(10.7)$ & $10(35.7)$ & $14(50)$ & $8.33 P<0.001$ \\
\hline $\mathrm{CRP}(\mathrm{mg} / \mathrm{L})$ & $0.40-2.87$ & $2.88-7.59$ & $7.60-17.23$ & $>17.23$ & \\
\hline Patients, $n$ & 34 & 35 & 35 & 34 & \\
\hline Mortality, $n$ (\% of total dead) & $3(10.7)$ & $1(3.6)$ & $10(35.7)$ & $14(50)$ & $20.45 P<0.001$ \\
\hline Albumin $(g / L)$ & $<39$ & $39-42$ & $42-45$ & $>45$ & \\
\hline Patients, $n$ & 36 & 38 & 37 & 32 & \\
\hline Mortality, $n$ (\% of total dead) & $17(60.7)$ & $2(7.1)$ & $6(21.5)$ & $3(10.7)$ & $27.60 P<0.001$ \\
\hline BNP (ng/L) & $<51.0$ & $51.0-116.8$ & $116.8-328.8$ & $>328.8$ & \\
\hline Patients, $n$ & 35 & 36 & 36 & 35 & \\
\hline Mortality, $n$ (\% of total dead) & $2(7.1)$ & $6(21.4)$ & $5(17.9)$ & $11(39.3)$ & $11.3 P=0.01$ \\
\hline NT-pro-BNP (ng/L) & $<206.5$ & $206.6-590.5$ & $590.6-1640.8$ & $>1640.8$ & \\
\hline Patients, $n$ & 35 & 36 & 36 & 35 & \\
\hline Mortality, $n$ (\% of total dead) & $1(3.6)$ & $5(17.9)$ & $7(25)$ & $15(53.6)$ & $19.50 P<0.001$ \\
\hline
\end{tabular}

BNP, B-type natriuretic peptide; CRP, C-reactive protein; NT-pro-BNP, N-terminal peptide pro-BNP; Tnl, troponin I; TnT, troponin T.

NT-pro-BNP (hazard ratio 1.571 , 95\% CI 1.151-2.143, $P=0.004)$ were most predictive of all the biomarkers for all-cause mortality.

Age at the beginning of the study, type of dialysis, the presence of any form of diabetes, the presence of insulin-dependent diabetes, the presence of documented hypertension, the presence of pre-existing cardiac comorbidities, having a history of a failed transplant, use of cardioprotective medications (angiotensin-converting enzyme inhibitors and beta-blockers) and time per session of dialysis were sequentially entered into the same Cox regression model in a forward conditional stepwise model. Albumin (hazard ratio 0.834, 95\% CI 0.779-0.893, $P<0.001)$ and the natural $\log$ transformed NT-pro-BNP (hazard ratio 1.585, 95\%CI 1.160-2.165, $P=0.004)$, still remained most predictive of all-cause mortality for each model. The Kaplan-Meier curve showing survival for the quartiles of NT-pro-BNP is shown in Figure 1.

Table 6 shows AUC calculations for the different variables. These show similar values as published, ${ }^{6}$ but our numbers are too small for meaningful assessment of cut points.

\section{Discussion}

Cardiovascular disease burden is disproportionately higher in the end-stage renal disease (ESRD) population. These patients commonly report less in the way of symptoms and in consequence may be treated less intensively. ${ }^{10-12}$ It is therefore of particular value to have biomarkers that can be used with relative ease to risk stratify these patients to allow for appropriate application of available treatment strategies.

Several biomarkers have been clearly documented to provide valuable information on patients at risk of developing significant cardiac disease. These include $\mathrm{TnT}^{3-5}$ and TnI, ${ }^{6,7} \mathrm{CRP}^{6}$ and also BNP ${ }^{13,14}$ and NT-pro-BNP. ${ }^{6}$ Our study supports the predictive value of all these biomarkers using univariate analysis. Our study is further validated by confirmation of previous observations namely that albumin is a powerful index of poor prognosis. ${ }^{9,15,16}$

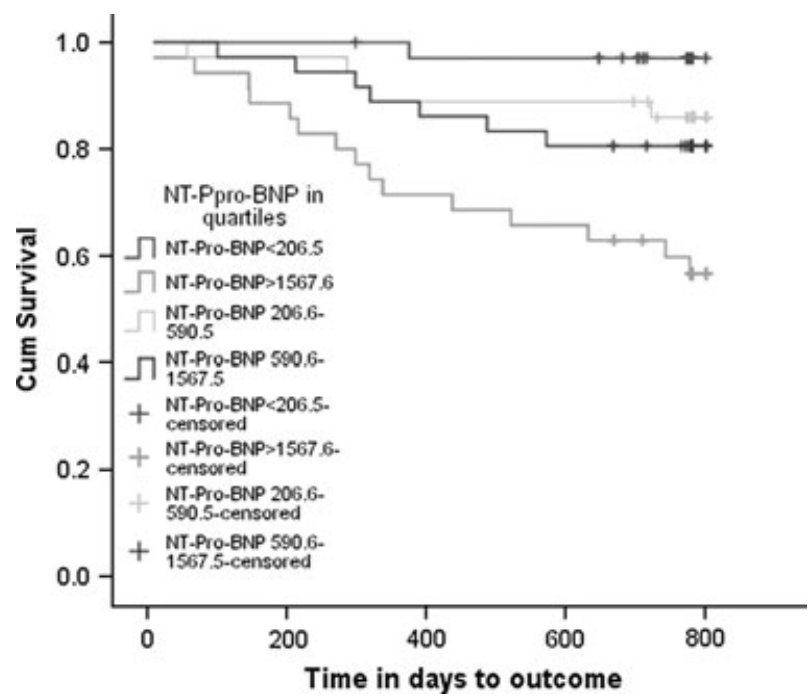

Figure 1 Kaplan-Meier survival functions for N-terminal peptide proBNP (NT-pro-BNP) in quartiles. 
Table 6 Area under the curve

\begin{tabular}{lccccc}
\hline $\begin{array}{l}\text { Test result } \\
\text { variables }\end{array}$ & Area & SE* & $\begin{array}{c}\text { Asymptotic } \\
\text { significance** }\end{array}$ & \multicolumn{2}{c}{$\begin{array}{c}\text { Asymptotic } \\
95 \% \mathrm{Cl}\end{array}$} \\
\hline NT-pro-BNP & 0.754 & 0.052 & 0.000 & 0.652 & 0.856 \\
BNP & 0.698 & 0.057 & 0.001 & 0.587 & 0.809 \\
Troponin I & 0.763 & 0.050 & 0.000 & 0.666 & 0.860 \\
Albumin level $^{\dagger}$ & 0.724 & 0.066 & 0.000 & 0.596 & 0.853 \\
CRP $_{\text {Troponin T }}$ & 0.719 & 0.055 & 0.000 & 0.610 & 0.828 \\
\hline
\end{tabular}

*Under the non-parametric assumption, ** null hypothesis: true area is 0.5 . †Smaller values of the test result variable indicate stronger evidence. BNP, B-type natriuretic peptide; $\mathrm{Cl}$, confidence interval; CRP, C-reactive protein; NT-pro-BNP, N-terminal peptide pro-BNP.

Although BNP, NT-pro-BNP, CRP and TnT have each been shown to be predictive of mortality in the dialysis population, ${ }^{6,8,9,13,17}$ ours is the first paper that provides measures of all these analytes coupled with a multivariate analysis. This has shown that NT-pro-BNP is the strongest predictor of mortality among the cardiac biomarkers studied. Additionally our data emphasized the importance of TnI as a predictor of mortality with similar hazard rate as TnT. This needs further study and may be a reflection of improved performance of newer assays in detecting TnI. ${ }^{7}$

There are several limitations to our study. The sample size and number of adverse events are relatively small and therefore the Cox regression analysis is restricted to a limited number of potential confounders. However, other studies measuring similar biomarkers provided similar outcomes. ${ }^{5,9,18}$ Our study only validates similar findings in a predominantly Caucasian, Australian population.

Although our population was relatively small, the probability that the Cox regression analysis results are a false positive is low. This probability can be calculated as the false-positive report probability (FPRP), ${ }^{19}$ or a 'crucial' Type I error, ${ }^{20,21}$ which is the probability that the null hypothesis is true when the null hypothesis is rejected. ${ }^{20}$ The magnitude of the FPRP is dependent on the prior probability $(\gamma)$ of a true association of the tested variable with a disease or condition; the $\alpha$-level or observed $P$-value; and the statistical power to detect the specified measure of the alternative hypothesis at the given $\alpha$-level or $P$-value. ${ }^{19-21}$ We estimate (data available on request) that the FPRP lies between 0.0044 and 0.04 .

Bias is unlikely to affect the internal validity of the results because of the completeness of the data collection and follow up. However, on a population basis, the use of one centre may provide a sample of patients different to the normal dialysis patient at other renal units. Although our results replicate that of Madsen et al., the external validity of the results needs further prospective confirmation for multivariate subgroup analysis. ${ }^{9}$

The reason for difference in predictive value of cardiac biomarkers in patients with ESRD is unclear, but may be a reflection of heterogeneity in cardiac pathology in these patients.

We did not have prospective echocardiographic data on all patients to allow for analysis of any relationship between components of ventricular structure and function and cardiac biomarkers. We were also unable to determine the influence of the findings of an echocardiogram on the predictive value of the cardiac biomarkers on mortality. However, another study measuring echocardiographic parameters in all the patients and with similar findings with respect to the NT-pro-BNP found no influence of the echocardiographic findings on all-cause mortality. ${ }^{9}$ Similarly in our study, echocardiographic parameters obtained from a subgroup of patients in our study $(n=99)$, who had had an echocardiogram as a clinical decision by the supervising physician, did not predict mortality nor alter the predictive outcome for NT-pro-BNP in a multivariate analysis using just this subgroup (data not shown).

Because the analysis is based on relatively small numbers both for our study and that of Madsen et al., further research is needed, preferably in collaborative multicentre studies with predetermined approximate samples size for inclusion of more potential confounding factors in the multivariate analysis. ${ }^{9}$ Also to assess the relative independent risk associated with any of the other biomarkers longer follow-up times are required. We plan to report our 4-year outcomes. Overall our results provide justification for use of health care resources for further research, but not for implementing our observations for clinical practice and screening.

In conclusion, it is becoming apparent that certain biomarkers are robust predictors of adverse outcomes in the dialysis population. What is desirable now is for the performance of multicentre larger scale observational studies to find the most robust prognostic predictors of all-cause and cardiovascular mortalities, with combined simultaneous cross-sectional biological and cardiac structural and functional investigations to investigate the causes of the changes in the biomarkers. Subsequently, randomized clinical trials of therapy studies can focus on the more relevant targets for intervention.

\section{References}

1 ANZDATA Registry Report, www.anzdata.org.au/anzdata/ AnzdataReport $/ 28^{\text {th }}$ Report/Ch03

2 Foley RN, Collins AJ. End stage renal disease in the United States: an update from the United States Renal Data System. J Am Soc Nephrol 2007; 18: 2644-48. 
3 Ooi DS, Veinot JP, Wells GA, House AA. Increased mortality in hemodialyzed patients with elevated serum troponin T: a one-year outcome study. Clin Biochem 1999; 32: 647-52.

4 Dierkes J, Domrose U, Westphal S, Ambrosch A, Bosselmann H-P, Neumann $\mathrm{H}$ et al. Cardiac troponin $\mathrm{T}$ predicts mortality in patients with end-stage renal disease. Circulation 2000; 102: 1964-9.

5 Khan NA, Hemmelgam BR, Tonelli M, Thompson CR, Levin A. Prognostic value of troponin T and I among asymptomatic patients with end stage renal disease: a meta-analysis. Circulation 2005; 112: 3088-96.

6 Apple FS, Murakami MM, Pearce LA, Herzog CA. Multi-biomarker risk stratification of $\mathrm{N}$-terminal pro-B-type natriuretic peptide, high-sensitivity C-reactive protein, and cardiac troponin $\mathrm{T}$ and $\mathrm{I}$ in end-stage renal disease for all-cause death. Clin Chem 2004; 50: 2279-85.

7 Hickman PE, Koerbin G, Southcott E, Tate J, Dimeski G, Carter A et al. Newer cardiac troponin I assays have similar performance to troponin $\mathrm{T}$ in patients with end-stage renal disease. Ann Clin Biochem 2007; 44: 285-9.

8 Sommerer C, Beimler J, Schwenger V, Heckele N, Katus HA, Giannitsis E et al. Cardiac biomarkers and survival in haemodialysis patients. Eur J Clin Invest 2007; 37: 350-56.

9 Madsen LH, Ladefoged S, Corell P, Schout M, Hildebrandt PR, Atar D. N-terminal pro brain natriuretic peptide predicts mortality in patients with end-stage renal disease in hemodialysis. Kidney Int 2007; 71: 548-54.

10 Wang TJ, Evans JC, Benjamin EJ, Levy D, LeRoy EC, Vasan RS. Natural history of asymptomatic left ventricular systolic dysfunction in the community. Circulation 2003a; 108: 977-82.

11 Wang TJ, Levy D, Benjamin EJ, Vasan RS. The epidemiology of 'asymptomatic' left ventricular systolic dysfunction: implications for screening. Ann Intern Med 2003b; 138: 907-16.

12 Zoccali C, Benedetto FA, Mallamaci F, Tripepi G, Giacone G, Cataliotti A et al. Prognostic value of echocardiographic indicators of left ventricular systolic function in asymptomatic dialysis patients. $J$ Am Soc Nephrol 2004; 15: 1029-37.

13 Zoccali C, Mallamaci F, Benedetto FA, Tripepi G, Parlongo S, Cataliotti A et al. Creed Investigators. Cardiac natriuretic peptides are related to left ventricular mass and function and predict mortality in dialysis patients. J Am Soc Nephrol 2001; 12: 1508-15.

14 Goto T, Takase H, Toriyama T, Sugiura T, Kurita Y, Tsuru $\mathrm{N}$ et al. Increased circulating levels of natriuretic peptides predict future cardiac event in patients with chronic hemodialysis. Nephron 2002; 92: 610-15.

15 Foley RN, Parfrey PS, Harnett JD, Kent GM, Murray DC, Barre PE. Hypoalbuminemia, cardiac morbidity. Mortality in end-stage renal disease. J Am Soc Nephrol 1996; 7: 728-36.

16 Cooper BA, Penne EL, Bartlett LH, Pollock CA. Protein malnutrition and hypoalbuminemia as predictors of vascular events and mortality in ESRD. Am J Kidney Dis 2004; 43: 61-6.

17 Satyan S, Light RP, Agarwal R. Relationships of $\mathrm{N}$-terminal pro-B-natriuretic peptide and cardiac troponin $\mathrm{T}$ to left ventricular mass and function and mortality in asymptomatic hemodialysis patients. Am J Kidney Dis 2007; 50: 1009-19.

18 Wang AY, Lam CW, Yu CM, Wang M, Chan IH, Zhang Y et al. N-terminal pro-brain natriuretic peptide: an independent risk predictor of cardiovascular congestion, mortality, and adverse cardiovascular outcomes in chronic peritoneal dialysis patients. J Am Soc Nephrol 2007; 18: 321-30.

19 Wacholder S, Chanock S, Garcia-Closas M, El Ghormli L, Rothman N. Assessing the probability that a positive report is false: an approach for molecular epidemiology studies. J Natl Cancer Inst 2004; 96: 434-42.

20 Maxwell SE, Kelley K, Rausch JJ. Sample Size Planning for Statistical Power and Accuracy in Parameter Estimation. Ann Rev Psychol 2008; 59: 537-63.

21 O'Brien RG, Castelloe JM. Sample size analysis for traditional hypothesis testing: concepts and issues. In: Dmitrienko A, Chuang-Stein C, D'Agostino R, eds. Pharmaceutical Statistics Using SAS: A Practical Guide, Cary, NC: SAS; 2007; 237-71. 